\title{
Advances in Gene Therapy for Movement Disorders
}

\author{
Hideki Mochizuki, ${ }^{\dagger \dagger}$ Toru Yasuda,* and M. Maral Mouradian ${ }^{*}$ \\ *Research Institute for Diseases of Old Age and ${ }^{\dagger}$ Department of Neurology, Juntendo University School of Medicine, 2-1-1 \\ Hongo, Bunkyo-ku, Tokyo 113-8421, Japan; ${ }^{\ddagger}$ Center for Neurodegenerative and Neuroimmunologic Diseases, Department of \\ Neurology, Robert Wood Johnson Medical School, University of Medicine and Dentistry of New Jersey, Piscataway, \\ New Jersey 08854
}

Summary: After nearly 20 years of preclinical experimentation with various gene delivery approaches in animal models of Parkinson's disease (PD), clinical trials are finally underway. The risk/benefit ratio for these procedures is now generally considered acceptable under approved protocols. The current vehicle for gene delivery to the human brain is recombinant adeno-associated viral vector, which is nonpathogenic and non-self-amplifying. Candidate genes tested in PD patients encode 1) glutamic acid decarboxylase, which is injected into the subthalamic nucleus to catalyze biosynthesis of the inhibitory neurotransmitter $\gamma$-aminobutyric acid and so essentially mimic deep brain stimulation of this nucleus; 2) aromatic Lamino acid decarboxylase, which converts L-dopa to dopamine; and 3) neurturin, a member of the glial cell line-derived neurotrophic factor family. Unraveling the genetic underpinnings of PD could allow gene therapy to go beyond modulating neurotransmission or providing trophic effects to dopaminergic neurons by delivering a specific missing or defective gene. For example, the parkin gene (PARK2) is linked to recessively inherited PD due to loss of function mutations; it prevents $\alpha$-synuclein-induced degeneration of nigral dopaminergic neurons in rats and nonhuman primates. On the other hand, for dominantly inherited Huntington's disease (HD), in which an expanded polyglutamine tract imparts to the protein huntingtin a toxic gain of function, repressing expression of the mutant allele in the striatum using RNA interference technology mitigates pathology and delays the phenotype in a mouse model. Here we review the current state of preclinical and clinical gene therapy studies conducted in PD and HD. Key Words: Movement disorders, neurodegeneration, Parkinson's disease, Huntington's disease, adeno-associated viral vectors, gene therapy, parkin protein, $\alpha$-synuclein.

\section{GENE THERAPY FOR PARKINSON'S DISEASE (PD)}

Since the early days of experimental gene therapy for other disorders, PD was considered an ideal brain disease for this approach, ${ }^{1}$ because of the relatively selective localization of the pathology to the substantia nigra (at least in the early stages of the disease), understanding dopamine biosynthesis and basal ganglia circuitry, knowledge of dopaminergic neurotrophic factors, and availability of animal models for proof-of-principle preclinical testing. In addition, the discovery of several genes responsible for inherited forms of PD renders the delivery of normal copies of some of these genes a rational strategy in targeted patient populations. Parallel with these developments in PD, improvements in vector

Address correspondence and reprint requests to: M. Maral Mouradian, M.D., UMDNJ-RWJMS, 683 Hoes Lane, Room 180, Piscataway, NJ 08854. E-mail: Mouradian@umdnj.edu. design have made it possible to deliver therapeutic genes directly into the brain with reasonable safety.

The two fundamental considerations in gene therapy for PD are what gene should be targeted and how to deliver that gene. For a genetic disorder in general, a rational strategy for gene-based therapy requires identification of the defective gene, elucidation of the functional properties of the protein product, and understanding of the mechanism by which the mutation alters the phenotype. In the case of $\mathrm{PD}$, advances in vectorology have fueled the development of clinical gene therapy protocols to augment dopaminergic neurotransmission, modulate basal ganglia circuitry, or preserve residual nigral neurons. The delivery of these ancillary genes in gene therapy protocols has preceded delivering disease-causing genes, which had to await their respective identification and the characterization of their protein biology.

\section{Gene delivery methods to the brain}

Gene therapy can be performed either in vivo, which involves direct introduction of a therapeutic gene into an 
appropriate brain region, or ex vivo, which involves transplantation of cells that are engineered in culture to express a therapeutic gene. The direct intracerebral injection is accomplished most efficiently and in a sustained manner using a viral vector. The ex vivo approach remains in a preclinical phase, because of safety considerations and the general poor survival of transplanted cells.

In vivo gene therapy. The recombinant adenoassociated virus (rAAV), which has advanced to clinical testing in PD patients, is a nonpathogenic parvovirus that can infect both dividing and nondividing cells, including neurons. This vector offers several advantages, including the fact that most of the wild-type viral genome is deleted and therefore it appears to have no significant toxicity. AAV vectors that are entirely free of helper viruses and do not encode any viral proteins are currently available. This is a significant improvement over other viral vectors, such as herpesvirus 1 (HSV-1) and adenovirus, which retain the ability to synthesize viral proteins. In addition, AAV integrates into a specific site on chromosome 19, permitting increased DNA stability and prolonged expression time. ${ }^{2}$ Minor disadvantages include lower viral titers than those obtained with adenovirus and small insert size; the latter is generally not an issue for most currently known candidate therapeutic genes for PD. Recent studies using several vectors derived from AAV serotypes (e.g., AAV1, -4, -5, and -6) have improved potency and broadened tropism. ${ }^{3}$

Cell vehicles for ex vivo gene transfer in PD. Ex vivo engineering of cells containing cDNAs encoding candidate therapeutic proteins is also being explored in animal models of PD. Early attempts at transplanting cell lines (e.g., fibroblasts, Schwann cells, myoblasts, neuroblastoma, glioma, and neuroendocrine cells) that were engineered with various candidate genes were quickly abandoned for gene transfer applications because they generally either form expanding tumors or are killed by host immune defenses. ${ }^{4,5}$ These failures stimulated the development of polymer-encapsulated cell technology to transplant xenogeneic cells engineered to secrete trophic factors. For example, striatal delivery of glial cell linederived neurotrophic factor (GDNF) via encapsulated cells can lead to sustained behavioral improvement in a bilateral rat model of $\mathrm{PD}^{6}$ and in 1-methyl-4-phenyl1,2,3,6-tetrahydropyridine (MPTP)-lesioned baboons. ${ }^{7}$

Another means of circumventing the limitations of grafted cell lines is the application of molecular techniques of conditional immortalization using nontransforming oncogenes such as the temperature-sensitive allele of the SV40 large tumor antigen. This manipulation allows cell growth at low permissive temperatures $\left(\sim 33^{\circ} \mathrm{C}\right)$ in culture, but not at the core temperature of $37^{\circ} \mathrm{C}$. Immortalization of rat fetal mesencephalic neurons using this method has resulted in behavioral recovery without immune rejection or tumor formation in 6hydroxydopamine (6-OHDA)-lesioned rats. ${ }^{8}$ Delivery of tyrosine hydroxylase (TH) cDNA using such cells increased L-dopa production in rodent and nonhuman primate models of PD. ${ }^{9}$ Alternatively, the use of cell lines that differentiate into neurons under appropriate conditions has also been tried. For example, PC12 cells engineered with nerve growth factor under the control of the zinc-inducible metallothionein promoter differentiate into neurons when grafted in the rat striatum. ${ }^{10}$

The idea of an autologous source of cells that are easily obtained from the patient and readily transduced by therapeutic genes in vitro has stimulated the use of primary skin fibroblasts as vehicles for gene transfer. ${ }^{11,12}$ Survival of these cells in the brain is not predictable, however. In addition, instead of integrating with host brain circuitry, they tend to displace the brain parenchyma by forming a globular clump of cells, which itself can disrupt rotational behavior in rodents. ${ }^{13}$ Astrocytes also potentially represent an autologous source of cell vehicles. ${ }^{14}$ They have been tested owing to their natural supportive role in the brain, their efficient secretory mechanisms, transducibility in vitro, and tendency to migrate from the graft site, thus minimizing mass effect. ${ }^{15,16}$ Astrocytic cells engineered with TH or GDNF and transplanted in the striatum ${ }^{17,18}$ or substantia nigra ${ }^{19}$ reportedly result in some behavioral recovery in the rat 6-OHDA model of PD.

Bone marrow stromal cells represent another autologous source of cell vehicles for gene therapy. ${ }^{20,21}$ These cells can be greatly expanded in vitro and induced to differentiate into multiple mesenchymal, glial, and neuronal cells. ${ }^{22-24}$ Marrow cells can also seed the brain in vivo and reside in the parenchyma as astrocytes, microglia, and neurons. ${ }^{25,26}$ Further, marrow-derived astrocytes tend to home in to the site of brain injury, such as ischemic stroke. ${ }^{27}$ These properties of marrow-derived brain cells have been exploited to deliver the GDNF cDNA in the mouse MPTP model, with resultant neuroprotection. $^{28}$

\section{Candidate therapeutic genes for PD}

Two categories of candidate therapeutic genes are currently being tested in gene therapy protocols in PD patients. The first are symptomatic molecules that relieve the motor manifestations of the disorder, such as dopamine biosynthetic enzymes or gene products that enhance an inhibitory neurotransmitter, without substantially influencing the underlying neurodegeneration. The second are restorative molecules that have the potential to retard the neurodegenerative process by one or more mechanisms.

Dopamine biosynthetic genes. Because $\mathrm{TH}$ is the rate-limiting enzyme in dopamine biosynthesis, attempts to deliver the cDNA encoding this protein have been a focus of intense investigation. ${ }^{29}$ One method of deliver- 
ing TH into the striatum is transplantation of a tissue that endogenously expresses $\mathrm{TH}$. The latter, in fact, has been one of the rationales for transplantation of human fetal mesencephalic tissue and carotid body autotransplants in patients with PD. ${ }^{30-33}$

Early approaches to delivering TH cDNA in the striatum of animal models of PD have included the in vivo injection of viral vectors HSV-1, AAV, and adenovirus. ${ }^{34-37}$ Ex vivo engineering of cell lines, primary cells, ${ }^{5,13,17}$ and immortalized cell lines generated from embryonic mesencephalic cultures have also been used in $e x$ vivo gene transfer studies, ${ }^{9}$ with various degrees of functional effects, none sufficient to advance to clinical testing.

Tyrosine hydroxylase is also critically dependent on the cofactor tetrahydrobiopterin (BH4) for its activity. The importance of $\mathrm{BH} 4$ has been demonstrated in fibroblast cell lines that do not produce sufficient amounts of $\mathrm{BH} 4$ to permit production of $\mathrm{L}$-dopa in vitro or in vivo. ${ }^{38}$ The addition of $\mathrm{BH} 4$ to these cells is necessary for $\mathrm{L}-$ dopa synthesis and behavioral recovery. Thus, BH4 must be either supplemented exogenously or by coexpressing guanine 5'-triphosphate cyclohydrolase (GTP-CH1), the rate-limiting enzyme in $\mathrm{BH} 4$ production. The expression of both TH and GTP-CH1 in fibroblasts grafted in denervated striata is reportedly required for the development of detectable basal L-dopa levels. ${ }^{13}$ In vivo microdialysis studies after gene transfer with AAV have shown that $\mathrm{TH}$ alone is not enough for $\mathrm{L}$-dopa production and that GTH-CH1 is required in the absence of exogenous BH4. ${ }^{39}$ Thus, the role of GTH-CH1 appears to apply to both in vivo and ex vivo gene transfer.

Another dopamine biosynthetic enzyme is aromatic L-amino acid decarboxylase (AADC), which converts L-dopa to dopamine. rAAV-mediated production of AADC in striatal cells was found to enhance conversion of endogenous L-dopa to dopamine and improve behavioral phenotype in a rat model of PD. ${ }^{40}$ Subsequently, a combination of striatal rAAV-AADC delivery and Ldopa administration in MPTP-lesioned nonhuman primates was found to result in long-term improvement in clinical rating scores, significantly lowered L-dopa requirements, and a reduction in L-dopa-induced side effects, compared with control vector and L-dopa treatment. ${ }^{41}$

Coadministration of two separate AAV vectors for TH and for AADC in the striatum of rats resulted in more efficient dopamine production and behavioral recovery than $\mathrm{TH}$ alone. ${ }^{42}$ Furthermore, in nonhuman primates that were rendered parkinsonian by MPTP administration, TH and AADC delivery in the striatum resulted in TH-positive cells and biochemical improvement, but no consistent behavioral improvement. ${ }^{43}$ Some reports, however, also suggest a detrimental effect of AADC on dopamine production, perhaps due to end-product inhibition of $\mathrm{TH}^{44}$ This effect might be cell-type-specific, perhaps seen in nonneuronal cells such as fibroblasts that cannot sequester dopamine into synaptic vesicles, thus allowing it to interact with and inhibit cytoplasmic TH. To circumvent the problem of end-product inhibition, constructs have been developed that have a truncation of the $\mathrm{N}$-terminal regulatory domain of TH, leaving only its catalytic domain. ${ }^{45}$

Genes to inhibit overactive neurons. Many of the motor manifestations of PD are believed to be due to disinhibition of the subthalamic nucleus (STN), leading to pathologic excitation of its target nuclei, the internal segment of the globus pallidus and substantia nigra pars reticulata. Silencing the excitatory glutamatergic neurons of the STN by overexpressing glutamic acid decarboxylase (GAD), the enzyme that catalyzes the synthesis of the inhibitory neurotransmitter $\gamma$-aminobutyric acid (GABA), by using an AAV vector, is another gene therapy approach being examined for PD. ${ }^{46}$ Thus, delivery of GAD cDNA in the STN may parallel the workings of deep brain stimulation. In the rat model, neurons transduced with such a vector produce mixed inhibitory responses associated with GABA release, successfully converting them into an inhibitory pathway and reversing parkinsonian behavioral phenotype. ${ }^{47}$ The effect of rAAV-GAD gene therapy has also been ascertained in macaque monkeys. ${ }^{48}$

Neuroprotective and neurorestorative genes. Because neurotrophic factors cannot cross the blood-brain barrier, and because of problems associated with intracerebroventricular delivery of these proteins, gene transfer approaches have been attempted in animal models of PD. To date, GDNF family proteins and brain-derived neurotrophic factor (BDNF) have been examined for this purpose. ${ }^{49,50}$ GDNF delivered in vivo with an adenoviral vector into the rat substantia nigra ${ }^{51}$ or striatum ${ }^{52,53}$ protected against subsequent 6-OHDA lesions. A similar approach of delivering GDNF also restored neural function even in rats with established 6-OHDA lesions. ${ }^{54}$ Adenovirus vector-mediated GDNF delivery was also effective in a mouse MPTP model. ${ }^{55}$ In addition, an AAV vector was used to deliver the GDNF cDNA to the rat substantia nigra and resulted in functional recovery. ${ }^{56}$

Neurturin is a member of the same protein family as GDNF and has similar pharmacological properties. Neurturin exerts potent actions on the survival and function of midbrain dopaminergic neurons ${ }^{57,58}$ and provides efficient neuroprotection of lesioned nigral neurons, similar to GDNF, when delivered by in vivo gene therapy. ${ }^{59}$ Using rAAV vector encoding neurturin (named CERE$120)$, Kordower and colleagues ${ }^{60-62}$ showed functional neuroprotection, neurorestoration, and enhanced activity of nigrostriatal dopamine system in MPTP-lesioned and in aged monkeys.

In addition, several potential cascades culminating in dopaminergic cell death in the substantia nigra have been described. Inhibiting such a cascade is one of the options 
for gene therapy in PD. One of these pathways is triggered by cytochrome c released from mitochondria, which promotes the activation of caspase 9 through apoptotic protease activating factor 1 (Apaf1). A recent study demonstrated that an AAV-derived Apaf1 dominant negative inhibitor (as an antiapoptotic gene therapy) prevented MPTP toxicity. ${ }^{63}$ In another study, adenoviral gene transfer of a protein caspase inhibitor, X-chromosome-linked inhibitor of apoptosis, also prevented MPTP-induced cell death of dopaminergic neurons in the substantia nigra of mice. ${ }^{64} \mathrm{~A}$ major issue in antiapoptotic gene therapy is the potential adverse effect of oncogenesis. Thus, transient expression of antiapoptotic molecules may be a better strategy than long-term expression.

Fibrillization and aggregation of $\alpha$-synuclein appears to play a critical role in PD. The chaperone heat-shock protein 70 (Hsp70) strongly inhibits $\alpha$-synuclein fibril formation via preferential binding to prefibrillar species. ${ }^{65}$ Several compounds can suppress the toxicity of $\alpha$-synuclein, such as $\beta$-synuclein, inhibitors of tissue transglutaminase, ${ }^{66}$ ribozymes, $\alpha$-synuclein siRNAi, ${ }^{67}$ and $\alpha$-synuclein antibody. ${ }^{68}$ Some of these also represent candidate genes suitable for neuroprotective gene therapy for PD.

A consistent neurobiological abnormality detected in parkinsonian substantia nigra is elevated oxidative stress and, therefore, efforts to minimize it can potentially have therapeutic value. The antioxidant enzyme glutathione peroxidase has neuroprotective capability. A viral vector carrying the human glutathione peroxidase 1 gene (GPXI) was reported to provide protection against 6-OHDA-induced neurotoxicity of dopaminergic neurons in the rat substantia nigra. ${ }^{69}$ This finding suggests the potential beneficial effect of gene therapy delivering antioxidant molecules.

\section{Transfer of disease genes}

Of the five PD-causing genes identified to date, two are dominantly inherited (SNCA, encoding $\alpha$-synuclein; $L R R K 2)$ and three are recessive (PARK2, encoding parkin; PARK7, alias $D J-1$; and PINK1, previously $P A R K 6) .{ }^{70}$ Gene therapy strategies, therefore, are aimed at delivering a normal copy of a recessive gene that has lost its physiologic function while also downregulating a dominant gene that has gained a toxic function. Thus, the three recessive PD genes are clear candidates for gene therapy in individuals who carry mutations in these respective genes. For example, in Park7-null mice (synonym: $D J-1$ ), restoration of Park7 expression via adenoviral vector mitigates the phenotype and enhances their resistance to MPTP-induced damage. ${ }^{71}$ In addition, these recessively inherited genes can conceivably be helpful as gene therapy candidates in sporadic PD, because sporadic cases share neuropathological and biochemical abnormalities including protein aggregation and oxidative stress-although the question as to whether the underly- ing pathophysiology is the same remains to be unanswered.

Prevention of neurodegeneration by PARK2 in animal models of PD. Clinical, genetic, and neuropathological observations indicate that excess $\alpha$-synuclein is toxic to dopaminergic neurons. In rats, rAAV-mediated overexpression of human wild-type SNCA causes progressive dopaminergic neurodegeneration. ${ }^{72}$ On the other hand, the major cause of autosomal recessive juvenile parkinsonism involves mutations in PARK2, the gene encoding parkin. ${ }^{73}$ Parkin is an E3 ubiquitin ligase that catalyzes polyubiquitination of unfolded or shortlived proteins, directing the substrates to proteasomal degradation. ${ }^{74}$ Accordingly, delivery of wild-type PARK2 is expected to provide therapeutic benefits in PARK2-type PD patients. In addition, oxidative posttranslational modifications of parkin-e.g., $S$-nitrosylation $^{75,76}$ or covalent binding of dopamine ${ }^{77}$-may be responsible for impairment of parkin activity in sporadic cases of PD. Such processes could lead to accumulation of substrate proteins, with subsequent dopaminergic neuronal neurodegeneration, as proposed in PARK2 PD patients.

$\alpha$-Synuclein is also known to accumulate significantly in the substantia nigra in normal aging. ${ }^{78,79}$ We reported previously that the rAAV-mediated overexpression of parkin ameliorates $\alpha$-synuclein-induced dopaminergic neurodegeneration in the substantia nigra in a rat model. ${ }^{80} \mathrm{An}$ other group has shown similar neuroprotection against A30P mutant SNCA using a lentiviral vector. ${ }^{81}$ The protective effect of $P A R K 2$ parkin gene therapy against $\alpha$-synuclein toxicity (governed by the SNCA gene) has also been shown in monkeys, with AAV vectors delivering both of these PD-associated genes. ${ }^{82}$ Hence, viral vector-mediated overexpression of parkin may have a neuroprotective effect against abnormally accumulated $\alpha$-synuclein, which is seen in both $\alpha$-synuclein-linked and sporadic PD patients.

Several groups have reported that parkin can rescue mitochondrial dysfunction and dopaminergic neurodegeneration caused by PINK1 defect in Drosophila models. ${ }^{83-85}$ Furthermore, viral vector-mediated PARK2 delivery prevents dopamine neuron loss induced by 6 OHDA and MPTP. ${ }^{86,87}$

These findings taken together suggest that PARK2 delivery may be therapeutically beneficial not only in familial PD patients but also in sporadic PD.

Preclinical study of PARK2 gene therapy in primates. We performed a preclinical study of PARK2 gene therapy in an $\alpha$-synuclein overexpression model of macaque monkeys (Macaca mulatta). We used serotype-1 rAAV (rAAV1) vector as a substitute for the serotype-2 rAAV (rAAV2) used in our previous studies. $^{72,80}$ Advantages for rAAV1 over rAAV2 include 
reduction of the time required to prepare high-titer viral stocks and the ability to express transgenes more rapidly.

First, we tried retrograde PARK2 gene delivery into the nigrostriatal dopaminergic neurons; i.e., rAAV1 vectors were injected into the putamen and retrogradely transported to dopaminergic cell bodies in the substantia nigra. We speculated that direct stereotaxic injections of viral vectors into the substantia nigra carries a high risk of surgical injury to other brain structures, whereas injections into the larger striatal tissue can be performed with greater certainty and safety. We expected this strategy to be valuable when neuroprotective molecules including parkin are supplied to dopamine neurons in the substantia nigra in PD patients.

We found that rAAV1 vectors showed not only anterograde but also retrograde transport of parkin (Fig. 1). Further, retrogradely transported parkin could protect against $\alpha$-synuclein-induced degeneration of dopamine nerve terminals. ${ }^{82}$ Unfortunately, the efficiency of retrograde transport was too low to be applicable for clinical trials. Currently, we are making an effort for direct introduction of rAAV1 vector into the substantia nigra. We have found a magnetic resonance imaging (MRI)-based navigation system (Medtronic, Minneapolis, MN) useful for accurately injecting viral vectors into deep brain structures.

This strategy has enabled us to generate an $\alpha$-synucleinoverexpression model with a progressive parkinsonian phenotype in macaque monkeys ( $M$. mulatta). In histological analyses, we found that the injection needle reached the substantia nigra precisely and confirmed foreign protein expression in nigral dopamine neurons. Therapeutic effects of parkin are currently being evaluated using this strategy. Possible negative effects of parkin overexpression in diseased and healthy neurons can be assessed carefully in these animals. These preclinical investigations can lead to clinical trials of disease genebased therapies, and can be a promising way for the treatment of familial and sporadic PD.

\section{Clinical gene therapy for PD}

Three gene therapy protocols have been approved and are ongoing currently.

Glutamic acid decarboxylase. Preclinical studies in rats and nonhuman primates have paved the way for the world's first FDA-approved gene therapy protocol for PD patients. ${ }^{88}$ An AAV vector carrying human GAD cDNA was infused into the STN of 12 patients with advanced PD in an open-label study. The center of the STN, which was localized with a stereotactic frame (Leksell, Stockholm, Sweden) and MRI guidance, was infused with vector solution at $0.5 \mu \mathrm{L}$ per minute over 100 minutes. Three dose levels were used in groups of four patients each.

Clinical assessments at 1 year revealed a statistically significant $27 \%$ improvement in motor function, as measured by the Unified Parkinson's Disease Rating Scale (UPDRS), on the side of the body that correlated with the treated side of the brain. The untreated side showed no significant improvement. Activities of daily living also showed a trend toward improvement. Furthermore, fluorodeoxyglucose positron emission tomography (PET) scans obtained at 1 year revealed that the treated side of the brain exhibited a significant reduction in thalamic metabolism but the untreated side showed further increase in abnormal metabolism. Notably, no adverse events related to the surgery or immune reaction to the virus were reported. ${ }^{88}$ These encouraging results need to be validated with a more rigorous trial design.

Aromatic L-amino acid decarboxylases. Preclinical studies suggest that the introduction of AADC cDNA has a potential to reduce the dose of L-dopa administration and delay or prevent the development of L-dopa associated motor complications. A phase I/II clinical trial of an AAV vector (AV201) containing the AADC cDNA was initiated at the University of California, San Francisco. and the Lawrence Berkeley National Laboratory in late $2004 .^{89}$ The vector is delivered directly to the striatum of patients with advanced PD, with the expectation
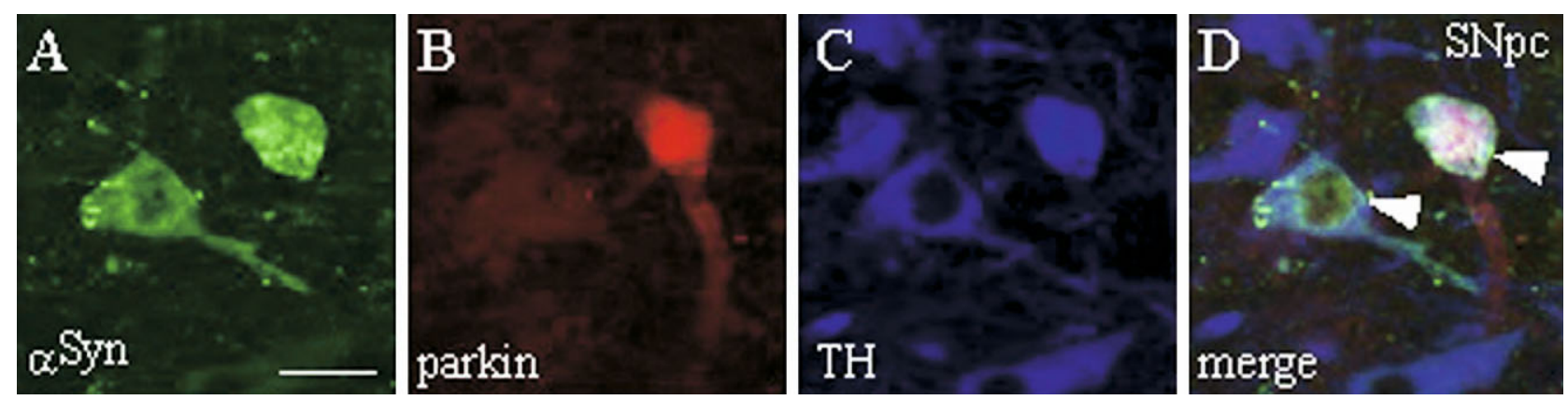

FIG. 1. Serotype-1 recombinant adeno-associated virus (rAAV) vector-mediated retrograde gene delivery in the primate brain. A cocktail of serotype-1 rAAV vectors each delivering a gene encoding $\alpha$-synuclein or parkin was injected into the putamen of a macaque monkey (Macaca mulatta). $\alpha$-Synuclein (A) and parkin (B) double-immunoreactive TH-positive (C) dopaminergic neurons were found in the substantia nigra pars compacta (SNpc), indicated by white arrowheads in the merged image (D). Scale bar: $25 \mu \mathrm{m}$. This figure was modified from Yasuda et al., 82 with permission. 
that they would respond more readily to L-dopa, because enhanced AADC expression should improve dopamine synthesis at the site of its action. The results from PET scans of a few patients obtained 6 months after AV201 infusion reportedly revealed increased activity of AADC in the targeted area of the brain, compared with the patients' pre-treatment scans. After clearance of safety issues, a phase II/III clinical trial is planned to detect the efficacy of this gene therapy approach for advanced PD. This approach is also being tested in a clinical protocol in Jichi Medical University in Japan.

Delivery of the neurturin gene into the striatum. In September 2005, a Phase I clinical trial to deliver the neurturin gene (NRTN, alias NTN) via CERE-120 in 12 PD patients was announced (http://www.ceregene.com/ press.asp, 09.21.05). The goal of this study was to determine the safety of this approach using standardized clinical assessments and brain imaging. ${ }^{90}$ In October 2006, Ceregene announced that none of the participants experienced serious adverse effects, and parkinsonian motor symptoms improved by $40 \%$. These promising results prompted the launch of a Phase II trial in which twothirds of the participants will receive active gene therapy and the rest will undergo sham surgery. ${ }^{91}$

Despite promising safety data from these early clinical trials, vigilance for possible adverse effects as a result of long-term transgene expression, as well as of the AAV vector itself, is paramount.

\section{GENE THERAPY FOR HUNTINGTON'S DISEASE}

Huntington's disease (HD) is a rare, dominantly inherited, neurodegenerative disorder caused by an expansion of a CAG repeat in the huntingtin gene (HTT) on chromosome 4 that is translated into expanded polyglutamine tract in the corresponding protein. The therapeutic promise of silencing mutant HTT expression was demonstrated in a tetracycline-regulated conditional mouse model of HD by Yamamoto et al..$^{92}$ Mice expressing a mutated huntingtin fragment demonstrated neuronal inclusions and progressive motor dysfunction. Blocking expression of mutant $\mathrm{Htt}$ in symptomatic mice led to disappearance of the inclusions and improvement of the behavioral changes. This promising observation suggests that gene therapy has the potential to be effective not only in preventing clinical disease but also in treating advanced stages of progressive neurodegenerative disorders.

\section{Therapeutic silencing of mutant huntingtin with SiRNA}

RNA interference (RNAi) has emerged as a potential therapeutic approach for neurodegenerative diseases, particularly those associated with autosomal dominant patterns of inheritance. In a preclinical study, Wang et al. ${ }^{93}$ used small, interfering RNAs (siRNAs) directed against the huntingtin gene to repress expression of transgenic mutant huntingtin in an HD mouse model. They showed that intraventricular injection of siRNAs at an early postnatal period inhibited transgenic huntingtin expression in neurons and resulted in decreased number and size of intranuclear inclusions in striatal neurons. In another study, AAV mediated delivery of short-hairpin RNA to Htt (siHUNT-1) in the striatum of HD mice significantly reduced expression of the pathogenic $\mathrm{Htt}$ allele, concomitant with a reduction in the size and number of neuronal intranuclear inclusions and partial normalization of neurotransmitter markers. ${ }^{94}$ Bilateral expression of rAAV5-siHUNT-1 resulted in delayed onset of motor deficits. These results suggest that a reduction in the level of striatal mutant $\mathrm{Htt}$ can ameliorate the HD phenotype in mice.

\section{Delivery of neurotrophic factor genes into the striatum in HD models}

Wild-type HTT upregulates transcription of BDNF, a pro-survival factor produced by cortical neurons that is necessary for survival of striatal neurons. Mutant HTT loses this beneficial activity, resulting in decreased production of cortical BDNF. ${ }^{95}$ Thus, delivery of the $B D N F$ gene has the potential to provide therapeutic efficacy in HD.

AAV-mediated gene transfer of BDNF into the striatum provides neuronal protection in a rodent model of HD. ${ }^{96}$ GDNF and neurturin are also neurotrophic factors expressed in the rat striatum during development and in the adult. Pérez-Navarro et al. ${ }^{97}$ reported that grafting a fibroblast cell line engineered to overexpress neurturin in the striatum protects striatal projection neurons but not interneurons in the quinolinate rat model of HD. Neurturin gene delivery by AAV vector also improves motor function and prevents striatal neuronal death in the 3-nitropropionic acid rat model of $\mathrm{HD} .^{98}$ Ceregene has already developed a clinical grade AAV-neurturin (CERE120), which is being tested in PD patients, so this therapy can be tested in HD patients as well.

\section{Gene therapy for upregulating neurogenesis}

Compared with control brains, HD brains manifest significant increase in cellular proliferation with evidence of generation of neuronal and glial cells in the subependymal layer adjacent to the caudate nucleus. The degree of cell proliferation increases with the severity of the disease pathology and the number of CAG repeats in $H T T .{ }^{99}$ However, these new immature neurons either die or differentiate into glial cells.

Ependymal overexpression of BDNF stimulates neurogenesis in the adult striatum from subependymal progenitor cells. The noggin protein potentiates this process by suppressing subependymal gliogenesis and thereby increasing progenitor availability. Cho et al. ${ }^{100}$ delivered 
both BDNF and noggin via adenoviral vector in HD mice and found increased neurogenesis, delayed onset of motor deficits, and longer survival than in empty vectorinjected control mice. This finding suggests that the beneficial effect of BDNF gene delivery in HD could be due to upregulation of neurogenesis in the striatum.

\section{Delivery of neurotrophic factor via encapsulated engineered cells}

Based on preclinical studies showing protective effect of ciliary neurotrophic factor (CNTF) on striatal neurons in animal models of HD, a phase I study in which polymer-encapsulated cells of a BHK cell line engineered to secrete CNTF were transplanted into the lateral ventricle of six HD patients. The capsules were replaced with new ones every 6 months over a 2 -year period. No sign of CNTF-induced toxicity was observed. Retrieved capsules contained variable numbers of surviving cells, and CNTF release was low in more than half the cases, which emphasizes the need for improving the technique. ${ }^{101}$ No follow-up information has been published on the clinical application of this approach in movement disorders.

\section{CHALLENGES OF GENE THERAPY}

In addition to the safety concerns related to possible oncogenesis and immunologic rejection that are considered serious in gene therapy protocols, an important aspect in the development of this novel therapeutic modality is the placebo effect of uncontrolled trials. This issue plagued studies of intracerebral GDNF infusion studies: the negative results of a placebo-controlled randomized trial ${ }^{102}$ contradicted the impressive results of a previous open-label uncontrolled trial. ${ }^{103}$ The placebo effect is a psychobiological phenomenon that can be attributed to different mechanisms. A PET study using the ability of endogenous dopamine to compete for $\left[{ }^{11} \mathrm{C}\right]$ raclopride binding demonstrated substantial dopamine release in response to placebo in PD patients. ${ }^{104}$ The strong placebo responses in PD patients who are implanted with electrodes for deep brain stimulation have been exploited by recording from single neurons after placebo treatment. These studies showed that placebo treatment reduces the activity of single neurons in the subthalamic nucleus in placebo-responsive patients. ${ }^{105}$ Thus, there is a need to monitor the placebo effect in gene therapy protocols.

Concerns about the placebo effect bring up the second problem, namely the ethics of sham surgery in a doubleblind gene therapy trial. Whereas the placebo-controlled design is the gold standard for evaluating new therapies, including gene therapy, the question of whether a surgical procedure to deliver an empty viral vector is safe enough to be used as a comparison group can be argued. A few studies involving PD patients have examined the risk of sham surgery in clinical trials. ${ }^{106,107}$ Although placebo surgeries were generally safe and well tolerated, the number of subjects who received these procedures was small. ${ }^{106}$ On the other hand, about half of researchers surveyed believed that an open-label efficacy trial would be unethical because it might lead to falsely positive results. ${ }^{107}$

In conclusion, judging from the phenomenal advances in experimental gene therapy for PD in the past few years, the future for this therapeutic modality looks more promising than ever-barring unforeseen hurdles in the early clinical trials.

Acknowledgments: This study was supported by the Program for Promotion of Fundamental Studies in Health Sciences of the National Institute of Biomedical Innovation (NIBIO) of Japan. M.M. Mouradian is the William Dow Lovett Professor of Neurology.

\section{REFERENCES}

1. Mouradian MM, Chase TN. Gene therapy for Parkinson's disease: current knowledge and future perspective. Gene Ther 1997; 4:504-506.

2. Linden RM, Ward P, Giraud C, Winocour E, Berns KI. Sitespecific integration by adeno-associated virus. Proc Natl Acad Sci U S A 1996;93:11288-11294.

3. Gao G, Vandenberghe LH, Wilson JM. New recombinant serotypes of AAV vectors. Curr Gene Ther 2005;5:285-297.

4. Freed WJ, Geller HM, Poltorak M, et al. Genetically altered and defined cell lines for transplantation in animal models of Parkinson's disease. Prog Brain Res 1990;82:11-21.

5. Wolff JA, Fisher LJ, Xu L, et al. Grafting fibroblasts genetically modified to produce L-dopa in a rat model of Parkinson disease. Proc Natl Acad Sci U S A 1989;86:9011-9014.

6. Sajadi A, Bensadoun JC, Schneider BL, Lo Bianco C, Aebischer P. Transient striatal delivery of GDNF via encapsulated cells leads to sustained behavioral improvement in a bilateral model of Parkinson disease. Neurobiol Dis 2006;22:119-129.

7. Kishima H, Poyot T, Bloch J, et al. Encapsulated GDNF-producing $\mathrm{C} 2 \mathrm{C} 12$ cells for Parkinson's disease: a pre-clinical study in chronic MPTP-treated baboons. Neurobiol Dis 2004;16:428439.

8. Prasad KN, Clarkson ED, La Rosa FG, Edwards-Prasad J, Freed CR. Efficacy of grafted immortalized dopamine neurons in an animal model of parkinsonism: a review. Mol Genet Metab 1998; 65:1-9.

9. Anton R, Kordower JH, Maidment NT, et al. Neural-targeted gene therapy for rodent and primate hemiparkinsonism. Exp Neurol 1994;127:207-218.

10. Rohrer DC, Nilaver G, Nipper V, Machida CA. Genetically modified PC12 brain grafts: Survivability and inducible nerve growth factor expression. Cell Transplant 1996;5:57-68.

11. Kawaja MD, Gage FH. Morphological and neurochemical features of cultured primary skin fibroblasts of Fischer 344 rats following striatal implantation. J Comp Neurol 1992;317:102116.

12. Lee WY, Lee EA, Jeon MY, Kang HY, Park YG. Vesicular monoamine transporter-2 and aromatic L-amino acid decarboxylase gene therapy prevents development of motor complications in parkinsonian rats after chronic intermittent L-3,4-dihydroxyphenylalanine administration. Exp Neurol 2006;197:215-224.

13. Bencsics C, Wachtel SR, Milstien S, Hatakeyama K, Becker JB, Kang UJ. Double transduction with GTP cyclohydrolase I and tyrosine hydroxylase is necessary for spontaneous synthesis of L-DOPA by primary fibroblasts. J Neurosci 1996;16:4449-4456. 
14. Ridet JL, Corti O, Pencalet P, et al. Toward autologous ex vivo gene therapy for the central nervous system with human adult astrocytes. Hum Gene Ther 1999;10:271-280.

15. Yoshimoto Y, Lin Q, Collier TJ, Frim DM, Breakefield XO, Bohn MC. Astrocytes retrovirally transduced with BDNF elicit behavioral improvement in a rat model of Parkinson's disease. Brain Res 1995;691:25-36.

16. Ljungberg MC, Stern G, Wilkin GP. Survival of genetically engineered, adult-derived rat astrocytes grafted into the 6-hydroxydopamine lesioned adult rat striatum. Brain Res 1999;816:29-37.

17. Lundberg C, Horellou P, Mallet J, Bjorklund A. Generation of DOPA-producing astrocytes by retroviral transduction of the human tyrosine hydroxylase gene: in vivo characterization and in vivo effects in the rat Parkinson model. Exp Neurol 1996;139: $39-53$.

18. Tornatore C, Baker-Cairns B, Yadid G, et al. Expression of tyrosine hydroxylase in an immortalized human fetal astrocyte cell line: in vitro characterization and engraftment into the rodent striatum. Cell Transplant 1996;5:145-163.

19. Ericson C, Georgievska B, Lundberg C. Ex vivo gene delivery of GDNF using primary astrocytes transduced with a lentiviral vector provides neuroprotection in a rat model of Parkinson's disease. Eur J Neurosci 2005;22:2755-2764.

20. Brazelton TR, Rossi FM, Keshet GI, Blau HM. From marrow to brain: expression of neuronal phenotypes in adult mice. Science 2000;290:1775-1779.

21. Nakano K, Migita M, Mochizuki H, Shimada T. Differentiation of transplanted bone marrow cells in the adult mouse brain. Transplantation 2001;71:1735-1740.

22. Azizi SA, Stokes D, Augelli BJ, DiGirolamo C, Prockop DJ. Engraftment and migration of human bone marrow stromal cells implanted in the brains of albino rats: similarities to astrocyte grafts. Proc Natl Acad Sci U S A 1998;95:3908-3913.

23. Munoz JR, Stoutenger BR, Robinson AP, Spees JL, Prockop DJ. Human stem/progenitor cells from bone marrow promote neurogenesis of endogenous neural stem cells in the hippocampus of mice [Erratum in: Proc Natl Acad Sci U S A 2006;103:2000-2]. Proc Natl Acad Sci U S A 2005;102:18171-18176.

24. Dezawa M, Kanno H, Hoshino M, et al. Specific induction of neuronal cells from bone marrow stromal cells and application for autologous transplantation. J Clin Invest 2004;113:1701-1710.

25. Eglitis MA, Mezey E. Hematopoietic cells differentiate into both microglia and macroglia in the brains of adult mice. Proc Natl Acad Sci U S A 1997;94:4080-4085.

26. Mezey E, Chandross KJ, Harta G, Maki RA, McKercher SR. Turning blood into brain: cells bearing neuronal antigens generated in vivo from bone marrow. Science 2000;290:1779-1782.

27. Eglitis MA, Dawson D, Park KW, Mouradian MM. Targeting of marrow-derived astrocytes to the ischemic brain. Neuroreport 1999;10:1289-1292.

28. Park KW, Eglitis MA, Mouradian MM. Protection of nigral neurons by GDNF-engineered marrow cell transplantation. Neurosci Res 2001;40:315-323.

29. Mouradian MM, Chase TN. Gene therapy of Parkinson's disease: An approach to the prevention or palliation of levodopa-associated motor complications. Exp Neurol 1997;144:51-57.

30. Espejo EF, Montoro RJ, Armengol JA, Lopez-Barneo J. Cellular and functional recovery of parkinsonian rats after intrastriatal transplantation of carotid body cell aggregates. Neuron 1998;20: 197-206.

31. Toledo-Aral JJ, Méndez-Ferrer S, Pardal R, Echevarría M, López-Barneo J. Trophic restoration of the nigrostriatal dopaminergic pathway in long-term carotid body-grafted parkinsonian rats. J Neurosci 2003;23:141-148.

32. Arjona V, Mínguez-Castellanos A, Montoro RJ, et al. Autotransplantation of human carotid body cell aggregates for treatment of Parkinson's disease. Neurosurgery 2003;53:321-328.

33. Mínguez-Castellanos A, Escamilla-Sevilla F, Hotton GR, et al. Carotid body autotransplantation in Parkinson disease: a clinical and positron emission tomography study. J Neurol Neurosurg Psychiatry 2007;78:825-831.
34. During MJ, Naegele JR, O’Malley KL, Geller AI. Long-term behavioral recovery in parkinsonian rats by an HSV vector expressing tyrosine hydroxylase. Science 1994;266:1399-1403.

35. Kaplitt MG, Leone P, Samulski RJ, et al. Long-term gene expression and phenotypic correction using adeno-associated virus vectors in the mammalian brain. Nat Genet 1994;8:148-154.

36. Rivière $\mathrm{C}$, Danos $\mathrm{O}$, Douar AM. Long-term expression and repeated administration of AAV type 1,2 and 5 vectors in skeletal muscle of immunocompetent adult mice. Gene Ther 2006;13: $1300-1308$.

37. Horellou P, Mallet J. Gene therapy for Parkinson's disease. Mol Neurobiol 1997;15:241-256.

38. Uchida K, Tsuzaki N, Nagatsu T, Kohsaka S. Tetrahydrobiopterin-dependent functional recovery in 6-hydroxydopaminetreated rats by intracerebral grafting of fibroblasts transfected with tyrosine hydroxylase cDNA. Dev Neurosci 1992;14:173180.

39. Mandel RJ, Rendahl KG, Spratt SK, Snyder RO, Cohen LK, Leff SE. Characterization of intrastriatal recombinant adeno-associated virus-mediated gene transfer of human tyrosine hydroxylase and human GTP-cyclohydrolase I in a rat model of Parkinson's disease. J Neurosci 1998;18:4271-4284.

40. Sánchez-Pernaute R, Harvey-White J, Cunningham J, Bankiewicz KS. Functional effect of adeno-associated virus mediated gene transfer of aromatic L-amino acid decarboxylase into the striatum of 6-OHDA-lesioned rats. Mol Ther 2001;4:324-330.

41. Bankiewicz KS, Forsayeth J, Eberling JL, et al. Long-term clinical improvement in MPTP-lesioned primates after gene therapy with AAV-hAADC. Mol Ther 2006;14:564-570.

42. Fan DS, Ogawa M, Fujimoto KI, et al. Behavioral recovery in 6-hydroxydopamine-lesioned rats by cotransduction of striatum with tyrosine hydroxylase and aromatic L-amino acid decarboxylase genes using two separate adeno-associated virus vectors. Hum Gene Ther 1998;9:2527-2535.

43. During MJ, Samulski RJ, Elsworth JD, et al. In vivo expression of therapeutic human genes for dopamine production in the caudates of MPTP-treated monkeys using an AAV vector. Gene Ther 1998;5:820-827.

44. Wachtel SR, Bencsics C, Kang UJ. Role of aromatic L-amino acid decarboxylase for dopamine replacement by genetically modified fibroblasts in a rat model of Parkinson's disease. J Neurochem 1997;9:2055-2063.

45. Moffat M, Harmon S, Haycock J, O’Malley KL. L-Dopa and dopamine-producing gene cassettes for gene therapy approaches to Parkinson's disease. Exp Neurol 1997;144:69-73.

46. During MJ, Kaplitt MG, Stern MB, Eidelberg D. Subthalamic GAD gene transfer in Parkinson disease patients who are candidates for deep brain stimulation. Hum Gene Ther 2001;12:1589 1591.

47. Luo J, Kaplitt MG, Fitzsimons HL, et al. Subthalamic GAD gene therapy in a Parkinson's disease rat model. Science 2002;298: 425-429.

48. Emborg ME, Carbon M, Holden JE, et al. Subthalamic glutamic acid decarboxylase gene therapy: changes in motor function and cortical metabolism. J Cereb Blood Flow Metab 2007;27:501509.

49. Olson L. The coming of age of the GDNF family and its receptors: gene delivery in a rat Parkinson model may have clinical implications. Trends Neurol Sci 1997;20:277-279.

50. Bohn MC. A commentary on glial cell line-derived neurotrophic factor (GDNF): from a glial secreted molecule to gene therapy. Biochem Pharmacol 1999;57:135-142.

51. Choi-Lundberg DL, Lin Q, Chang YN, et al. Dopaminergic neurons protected from degeneration by GDNF gene therapy. Science 1997;75:838-841.

52. Bilang-Bleuel A, Revah F, Colin P, et al. Intrastriatal injection of an adenoviral vector expressing glial-cell-line-derived neurotrophic factor prevents dopaminergic neuron degeneration and behavioral impairment in a rat model of Parkinson's disease. Proc Natl Acad Sci U S A 1997;94:8818-8823.

53. Choi-Lundberg DL, Lin Q, Schallert T, et al. Behavioral and cellular protection of rat dopaminergic neurons by an adenoviral 
vector encoding glial cell line-derived neurotrophic factor. Exp Neurol 1998;154:261-275.

54. Lapchak PA, Araujo DM, Hilt DC, Sheng J, Jiao S. Adenoviral vector-mediated GDNF gene therapy in a rodent lesion model of late stage Parkinson's disease. Brain Res 1997;777:153-160.

55. Kojima H, Abiru Y, Sakajiri K, et al. Adenovirus-mediated transduction with human glial cell line-derived neurotrophic factor gene prevents 1-methyl-4-phenyl-1,2,3,6-tetrahydropyridine-induced dopamine depletion in striatum of mouse brain. Biochem Biophys Res Commun 1997;238:569-573.

56. Mandel RJ, Spratt SK, Snyder RO, Leff SE. Midbrain injection of recombinant adeno-associated virus encoding rat glial cell linederived neurotrophic factor protects nigral neurons in a progressive 6-hydroxydopamine-induced degeneration model of Parkinson's disease in rats. Proc Natl Acad Sci U S A 1997;94:14083-14088.

57. Kotzbauer PT, Lampe PA, Heuckeroth RO, et al. Neurturin, a relative of glial-cell-line-derived neurotrophic factor. Nature 1996;384:467-470.

58. Horger BA, Nishimura MC, Armanini MP, et al. Neurturin exerts potent actions on survival and function of midbrain dopaminergic neurons. J Neurosci 1998;18:4929-4937.

59. Rosenblad C, Kirik D, Devaux B, Moffat B, Phillips HS, Björklund $\mathrm{A}$. Protection and regeneration of nigral dopaminergic neurons by neurturin or GDNF in a partial lesion model of Parkinson's disease after administration into the striatum or the lateral ventricle. Eur J Neurosci 1999;11:1554-66.

60. Kordower JH, Herzog CD, Dass B, et al. Delivery of neurturin by AAV2 (CERE-120)-mediated gene transfer provides structural and functional neuroprotection and neurorestoration in MPTPtreated monkeys. Ann Neurol 2006;60:706-715.

61. Herzog CD, Dass B, Holden JE, et al. Striatal delivery of CERE120 , an AAV2 vector encoding human neurturin, enhances activity of the dopaminergic nigrostriatal system in aged monkeys. Mov Disord 2007;22:1124-1132.

62. Gasmi M, Brandon EP, Herzog CD, et al. AAV2-mediated delivery of human neurturin to the rat nigrostriatal system: longterm efficacy and tolerability of CERE-120 for Parkinson's disease. Neurobiol Dis 2007;27:67-76.

63. Mochizuki H, Hayakawa H, Migita M, et al. An AAV-derived Apaf-1 dominant negative inhibitor prevents MPTP toxicity as antiapoptotic gene therapy for Parkinson's disease. Proc Natl Acad Sci U S A 2001;98:10918-10923.

64. Eberhardt O, Coelln RV, Kugler S, et al. Protection by synergistic effects of adenovirus-mediated X-chromosome-linked inhibitor of apoptosis and glial cell line-derived neurotrophic factor gene transfer in the 1-methyl-4-phenyl-1,2,3,6-tetrahydropyridine model of Parkinson's disease. J Neurosci 2000;20:9126-9134.

65. Dedmon MM, Christodoulou J, Wilson MR, Dobson CM. Heat shock protein 70 inhibits $\alpha$-synuclein fibril formation via preferential binding to prefibrillar species. J Biol Chem 2005;280: 14733-14740.

66. Junn E, Ronchetti RD, Quezado MM, Kim SY, Mouradian MM. Tissue transglutaminase-induced aggregation of $\alpha$-synuclein: implications for Lewy body formation in Parkinson's disease and dementia with Lewy bodies. Proc Natl Acad Sci U S A 2003; 100:2047-2052.

67. Hayashita-Kinoh H, Yamada M, Yokota T, Mizuno Y, Mochizuki $H$. Down-regulation of $\alpha$-synuclein expression can rescue dopaminergic cells from cell death in the substantia nigra of Parkinson's disease rat model. Biochem Biophys Res Commun 2006; 341:1088-1095.

68. Masliah E, Rockenstein E, Adame A, et al. Effects of $\alpha$-synuclein immunization in a mouse model of Parkinson's disease. Neuron 2005; 46:857-868

69. Ridet JL, Bensadoun JC, Déglon N, Aebischer P, Zurn AD. Lentivirus-mediated expression of glutathione peroxidase: neuroprotection in murine models of Parkinson's disease. Neurobiol Dis 2006;21:29-34.

70. Farrer MJ. Genetics of Parkinson disease: paradigm shifts and future prospects. Nat Rev Genet 2006;7:306-318.

71. Kim RH, Smith PD, Aleyasin H, et al. Hypersensitivity of DJ-1deficient mice to 1-methyl-4-phenyl-1,2,3,6-tetrahydropyrindine
(MPTP) and oxidative stress. Proc Natl Acad Sci U S A 2005; 102:5215-5220.

72. Yamada M, Iwatsubo T, Mizuno Y, Mochizuki H. Overexpression of $\alpha$-synuclein in rat substantia nigra results in loss of dopaminergic neurons, phosphorylation of $\alpha$-synuclein and activation of caspase-9: resemblance to pathogenetic changes in Parkinson's disease. J Neurochem 2004;91:451-461.

73. Kitada T, Asakawa S, Hattori N, et al. Mutations in the parkin gene cause autosomal recessive juvenile parkinsonism. Nature 1998;392:605-608

74. Shimura H, Hattori N, Kubo S, et al. Familial Parkinson disease gene product, parkin, is a ubiquitin-protein ligase. Nat Genet 2000;25:302-305.

75. Chung KK, Thomas B, Li X, et al. S-nitrosylation of parkin regulates ubiquitination and compromises parkin's protective function. Science 2004;304:1328-1331.

76. Yao D, Gu Z, Nakamura T, et al. Nitrosative stress linked to sporadic Parkinson's disease: S-nitrosylation of parkin regulates its E3 ubiquitin ligase activity [Erratum in: Proc Natl Acad Sci U S A 2004;101:13969]. Proc Natl Acad Sci U S A 2004;101: 10810-10814.

77. LaVoie MJ, Ostaszewski BL, Weihofen A, Schlossmacher MG, Selkoe DJ. Dopamine covalently modifies and functionally inactivates parkin. Nat Med 2005;11:1214-1221.

78. Li W, Lesuisse C, Xu Y, Troncoso JC, Price DL, Lee MK. Stabilization of $\alpha$-synuclein protein with aging and familial Parkinson's disease-linked A53T mutation. J Neurosci 2004;24: 7400-7409.

79. Chu Y, Kordower JH. Age-associated increases of $\alpha$-synuclein in monkeys and humans are associated with nigrostriatal dopamine depletion: Is this the target for Parkinson's disease? Neurobiol Dis 2007;25:134-149.

80. Yamada M, Mizuno Y, Mochizuki H. Parkin gene therapy for $\alpha$-synucleinopathy: a rat model of Parkinson's disease. Hum Gene Ther 2005;16:262-270.

81. Lo Bianco C, Schneider BL, Bauer M, et al. Lentiviral vector delivery of parkin prevents dopaminergic degeneration in an $\alpha$-synuclein rat model of Parkinson's disease. Proc Natl Acad Sci U S A 2004:101:17510-17515.

82. Yasuda T, Miyachi S, Kitagawa R, et al. Neuronal specificity of $\alpha$-synuclein toxicity and effect of parkin co-expression in primates. Neuroscience 2007;144:743-753.

83. Park J, Lee SB, Lee S, et al. Mitochondrial dysfunction in Drosophila PINK1 mutants is complemented by parkin. Nature 2006 441:1157-1161.

84. Clark IE, Dodson MW, Jiang C, et al. Drosophila PINK1 is required for mitochondrial function and interacts genetically with parkin. Nature 2006;441:1162-1166.

85. Yang Y, Gehrke S, Imai Y, et al. Mitochondrial pathology and muscle and dopaminergic neuron degeneration caused by inactivation of Drosophila Pink1 is rescued by parkin. Proc Natl Acad Sci U S A 2006;103:10793-10798.

86. Vercammen L, Van der Perren A, Vaudano E, et al. Parkin protects against neurotoxicity in the 6-hydroxydopamine rat model for Parkinson's disease. Mol Ther 2006;14:716-723.

87. Paterna JC, Leng A, Weber E, Feldon J, Bueler H. DJ-1 and parkin modulate dopamine-dependent behavior and inhibit MPTP-induced nigral dopamine neuron loss in mice [Erratum in: Mol Ther 2007;15:1221]. Mol Ther 2007;15:698-704.

88. Kaplitt MG, Feigin A, Tang C, et al. Safety and tolerability of gene therapy with an adeno-associated virus (AAV) borne GAD gene for Parkinson's disease: an open label, phase I trial. Lancet 2007;369:2097-2105.

89. Mandel RJ, Burger C. Clinical trials in neurological disorders using AAV vectors: promises and challenges. Curr Opin Mol Ther 2004;6:482-490.

90. Fjord-Larsen L, Johansen JL, Kusk P, et al. Efficient in vivo protection of nigral dopaminergic neurons by lentiviral gene transfer of a modified neurturin construct. Exp Neurol 2005;195: 49-60.

91. Check E. Second chance. Nat Med 2007;13:770-771. 
92. Yamamoto A, Lucas JJ, Hen R. Reversal of neuropathology and motor dysfunction in a conditional model of Huntington's disease. Cell 2000;101:57-66.

93. Wang YL, Liu W, Wada E, Murata M, Wada K, Kanazawa I. Clinico-pathological rescue of a model mouse of Huntington's disease by siRNA. Neurosci Res 2005;53:241-249.

94. Rodriguez-Lebron E, Denovan-Wright EM, Nash K, Lewin AS, Mandel RJ. Intrastriatal rAAV-mediated delivery of anti-huntingtin shRNAs induces partial reversal of disease progression in R6/1 Huntington's disease transgenic mice. Mol Ther 2005;12: 618-633.

95. Zuccato C, Ciammola A, Rigamonti D, et al. Loss of huntingtinmediated BDNF gene transcription in Huntington's disease. Science 2001;293:493-498.

96. Kells AP, Fong DM, Dragunow M, During MJ, Young D, Connor B. AAV-mediated gene delivery of BDNF or GDNF is neuroprotective in a model of Huntington disease. Mol Ther 2004;9:682688.

97. Pérez-Navarro E, Akerud P, Marco S, et al. Neurturin protects striatal projection neurons but not interneurons in a rat model of Huntington's disease. Neuroscience 2000;98:89-96.

98. Ramaswamy S, McBride JL, Herzog CD, et al. Neurturin gene therapy improves motor function and prevents death of striatal neurons in a 3-nitropropionic acid rat model of Huntington's disease. Neurobiol Dis 2007;26:375-384.

99. Curtis MA, Penney EB, Pearson AG, et al. Increased cell proliferation and neurogenesis in the adult human Huntington's disease brain. Proc Natl Acad Sci U S A 2003;100:9023-9027.

100. Cho SR, Benraiss A, Chmielnicki E, Samdani A, Economides A,
Goldman SA. Induction of neostriatal neurogenesis slows disease progression in a transgenic murine model of Huntington disease. J Clin Invest 2007;117:2889-2902.

101. Bloch J, Bachoud-Levi AC, Deglon N, et al. Neuroprotective gene therapy for Huntington's disease, using polymer-encapsulated cells engineered to secrete human ciliary neurotrophic factor: results of a phase I study. Hum Gene Ther 2004;15: 968-975.

102. Lang AE, Gill S, Patel NK, et al. Randomized controlled trial of intraputamenal glial cell line-derived neurotrophic factor infusion in Parkinson disease [Erratum in: Ann Neurol 2006;60:747]. Ann Neurol 2006;59:459-466.

103. Gill SS, Patel NK, Hotton GR, et al. Direct brain infusion of glial cell line-derived neurotrophic factor in Parkinson disease. Nat Med 2003;9:589-595.

104. de la Fuente-Fernández R, Ruth TJ, Sossi V, Schulzer M, Calne DB, Stoessl AJ. Expectation and dopamine release: mechanism of the placebo effect in Parkinson's disease. Science 2001;293: 1164-1166.

105. Benedetti F, Colloca L, Torre E, et al. Placebo-responsive Parkinson patients show decreased activity in single neurons of subthalamic nucleus. Nat Neurosci 2004;7:587-588.

106. Frank S, Kieburtz K, Holloway R, Kim SY. What is the risk of sham surgery in Parkinson disease clinical trials? A review of published reports. Neurology 2005;65:1101-1103.

107. Kim SY, Frank S, Holloway R, Zimmerman C, Wilson R, Kieburtz K. Science and ethics of sham surgery: a survey of Parkinson disease clinical researchers. Arch Neurol 2005;62: 1357-1360. 